\title{
In situTEM modification of individual silicon nanowires and their charge transport mechanisms
}

Alam, Sardar B.; Andersen, Christopher Røhl; Panciera, Federico; Nilausen, Aage A.S.; Hansen, Ole; Ross, Frances M.; Mølhave, Kristian

Published in:

Nanotechnology

Link to article, DOI:

$10.1088 / 1361-6528 / a b a b c 8$

Publication date:

2020

Document Version

Peer reviewed version

Link back to DTU Orbit

Citation $(A P A)$ :

Alam, S. B., Andersen, C. R., Panciera, F., Nilausen, A. A. S., Hansen, O., Ross, F. M., \& Mølhave, K. (2020). In situTEM modification of individual silicon nanowires and their charge transport mechanisms. Nanotechnology, 31, [494002]. https://doi.org/10.1088/1361-6528/ababc8

\section{General rights}

Copyright and moral rights for the publications made accessible in the public portal are retained by the authors and/or other copyright owners and it is a condition of accessing publications that users recognise and abide by the legal requirements associated with these rights.

- Users may download and print one copy of any publication from the public portal for the purpose of private study or research.

- You may not further distribute the material or use it for any profit-making activity or commercial gain

- You may freely distribute the URL identifying the publication in the public portal 


\title{
In situ TEM modification of individual silicon nanowires and their charge transport mechanisms
}

\author{
Sardar B. Alam ${ }^{1,0}$, Christopher R. Andersen ${ }^{1,0}$, Federico Panciera2-4, Aage A. S. \\ Nilausen ${ }^{1}$, Ole Hansen ${ }^{1}$, Frances M. Ross4, 5 and Kristian Mølhave1 \\ 1. National Centre for Nano Fabrication and Characterization, Technical University of Denmark, 2800 \\ Kgs. Lyngby, Denmark \\ 2. University of Paris-Saclay, CNRS, Centre for Nanoscience and Nanotechnology, 91120, Palaiseau, \\ France \\ 3. Department of Engineering, University of Cambridge, Cambridge, United Kingdom \\ 4. IBM T. J. Watson Research Center, Yorktown Heights, NY, United States \\ 5. Department of Materials Science and Engineering, Massachusetts Institute of Technology, Cambridge, \\ Massachusetts, USA, United States
}

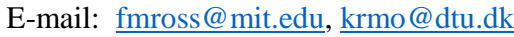

Received xxxxxx

Accepted for publication $\mathrm{xxxxxx}$

Published xxxxxx

\begin{abstract}
Correlating the structure and composition of nanowires grown by the vapour-liquid-solid (VLS) mechanism with their electrical properties is essential for designing nanowire devices. In situ transmission electron microscopy (TEM) that can image while simultaneously measuring the current-voltage $(I-V)$ characteristics of individual isolated nanowires is a unique tool for linking changes in structure with electronic transport. Here we grow and electrically connect silicon nanowires inside a TEM to perform in situ electrical measurements on individual nanowires both at high temperature and upon surface oxidation, as well as under ambient conditions. As-grown, the oxide-free nanowires have nonlinear $I-V$ characteristics. We analyse the $I-V$ measurements in terms of both bulk and injection limited transport models, finding Joule heating effects, bulk-limiting effects for thin nanowires and an injection-limiting effect for thick wires when high voltages are applied. When the nanowire surface is modified by in situ oxidation, drastic changes occur in the electronic properties. We investigate the relation between the observed geometry, changes in the surface structure and changes in electronic transport, obtaining information for individual nanowires that is inaccessible to other measuring techniques.
\end{abstract}

Keywords: nanowire, electronic transport, in situ measurements, TEM, surface oxidation

\section{Introduction}

The electronic properties of nanowires grown by the vapour-liquid-solid (VLS) mechanism are sensitive to fine details of the nanowire composition, structure and surface configuration. For a wide range of VLS nanowire applications, for example in logic [1,2], solar cells [3,4], optoelectronics [5-
7], and biological [8,9], and chemical [10-14] sensing [1517], achieving the required device performance depends on careful consideration of nanowire composition and surface structure and the interface between the nanowire and the rest of the device. However, determining the structural details of individual nanowires, such as the presence of defects and the surface condition, and relating this to the electronic properties after device fabrication, is a challenging task. In situ 
transmission electron microscopy (TEM) has been a vital tool for visualising and understanding the physical processes involved in the growth of nanowires by the VLS mechanism [18]. Furthermore, in situ TEM has also been able to probe the electrical properties of nanowires inside the TEM while simultaneously recording the structure at high spatial resolution [19-21]. Such in situ measurements can help us develop nanodevices based on functional nanowires by providing an improved foundation for rational design. Direct correlation of the composition, structure and electrical characteristics of the nanowire component of a device is not straightforward using other measurement techniques [22]. Instead, in situ TEM measurements on pristine and processed nanowires will increasingly allow us to select nanowire parameters, for example by tuning the growth conditions or subsequently modifying the nanowire surface, that are best suited for optimal performance in a given device architecture.

In this work, we have grown and electrically characterised gold-catalysed silicon nanowires inside an in situ TEM that has ultra-high vacuum (UHV) at the sample area. We used microcantilever based samples that allow us to grow individual nanowires in a configuration where they are electrically connected at the two ends (Figure S1). Figure 1(a) shows schematics of a sample. A microfabricated chip with Joule heated monocrystalline silicon cantilevers [23] forms the substrate for epitaxial growth of the nanowires. Such chips have been used to study the creation of silicon nanowire devices [19] as well as migration [24] and high electric field phenomena [25]. Here, the nanowires are grown between two adjacent but electrically isolated microcantilever heaters to form a suspended bridging nanowire [24] (Figure 1(b)). This configuration introduces a circuit with resistances in series from the two cantilevers, the bridging nanowire and the two nanowire-to-cantilever spreading resistances (Figure S2). With this setup, we can measure the $I-V$ characteristics of the as-grown nanowire under vacuum at room temperature and higher temperatures. We can also introduce oxygen into the sample region of the TEM to study the influence on transport of having oxidised the surface. The experimental setup is described in more detail in the Supporting Information.

Our in situ measurements show that nanowires exhibit nonlinear $I$ - $V$ curves (Figure 1(c)) that depend on the nanowire diameter and surface chemistry. In the following discussion, we introduce a range of nonlinear transport models before analysing our data and assigning the model that best fits the in situ measurements.

\section{Transport models for nanowires}

\subsection{Ohmic behaviour and Joule heating}

At low voltages, a semiconductor will typically follow an Ohmic behaviour where the $I-V$ curve is linear. One source of nonlinearity in $I-V$ data is self-heating, in which the increase in temperature due to Joule heating changes the electrical resistance. A temperature increase will decrease the resistance of an intrinsic semiconductor, typically with a strongly nonlinear temperature coefficient of resistivity (TCO). The resistance of an extrinsic (doped) semiconductor, over a limited temperature range, will vary linearly with temperature (positive or negative TCO); but at higher temperatures, it may behave as an intrinsic semiconductor. Since nanowires in this work are suspended, resistive self-heating could lead to considerable temperature increase.

A model combining Ohmic conductance with resistive heating has been described by Mølhave et al. [26]. As a rough approximation, we may assume a parabolic temperature profile along the length of the nanowire with peak temperature in the center. Resistive heating of the nanowire can lead to linear dependence of either resistance or conductivity with temperature. A linear resistance dependence leads to a nonlinear $I-V$ curve, where a linear plot can be made of $V / I$ vs. power $I V$, while a linear conductivity dependence will give a linear plot of $I / V$ vs. $I V$ - as seen, for example, in carbon nanotubes [26].

Several other possible transport mechanisms can also lead to nonlinear $I-V$ characteristics. These involve bulk-limited models including space charge limited current (SCLC) and the Poole-Frenkel (PF) effect, and injection limited models of Schottky and Fowler-Nordheim (FN) effects. These models are illustrated in Figure 1(d)-(g) and summarised in Table 1.

\subsection{Bulk-limited current models}

SCLC dominates when the injected charge density is higher than the thermal equilibrium density of carriers [27]. All injected electrons (or holes) are then transported via the conduction (or valence) band. This is typical for insulators, wide-gap semiconductors and poorly conducting polymers [28]. The SCLC $I-V$ relations can be divided into three regimes [27]:

- A low bias Ohmic regime $(I \propto V)$

- A high bias SCLC regime following a power law $(I \propto$ $\left.V^{a}\right)$, with exponent $a=2$, when the material is trap-free as formulated by Mott-Gurney [29]. If the material has traps with an exponential distribution of energies as described by the Mark-Helfrich equation [30], $a>2$; this situation is illustrated in Figure 1(d). The injected carriers will then be partly consumed to fill up the trap levels $\left(E_{t}\right)$, which lowers the current.

- An intermediate voltage regime between the two with an exponential increase near the trap-filled limit voltage [27]. 


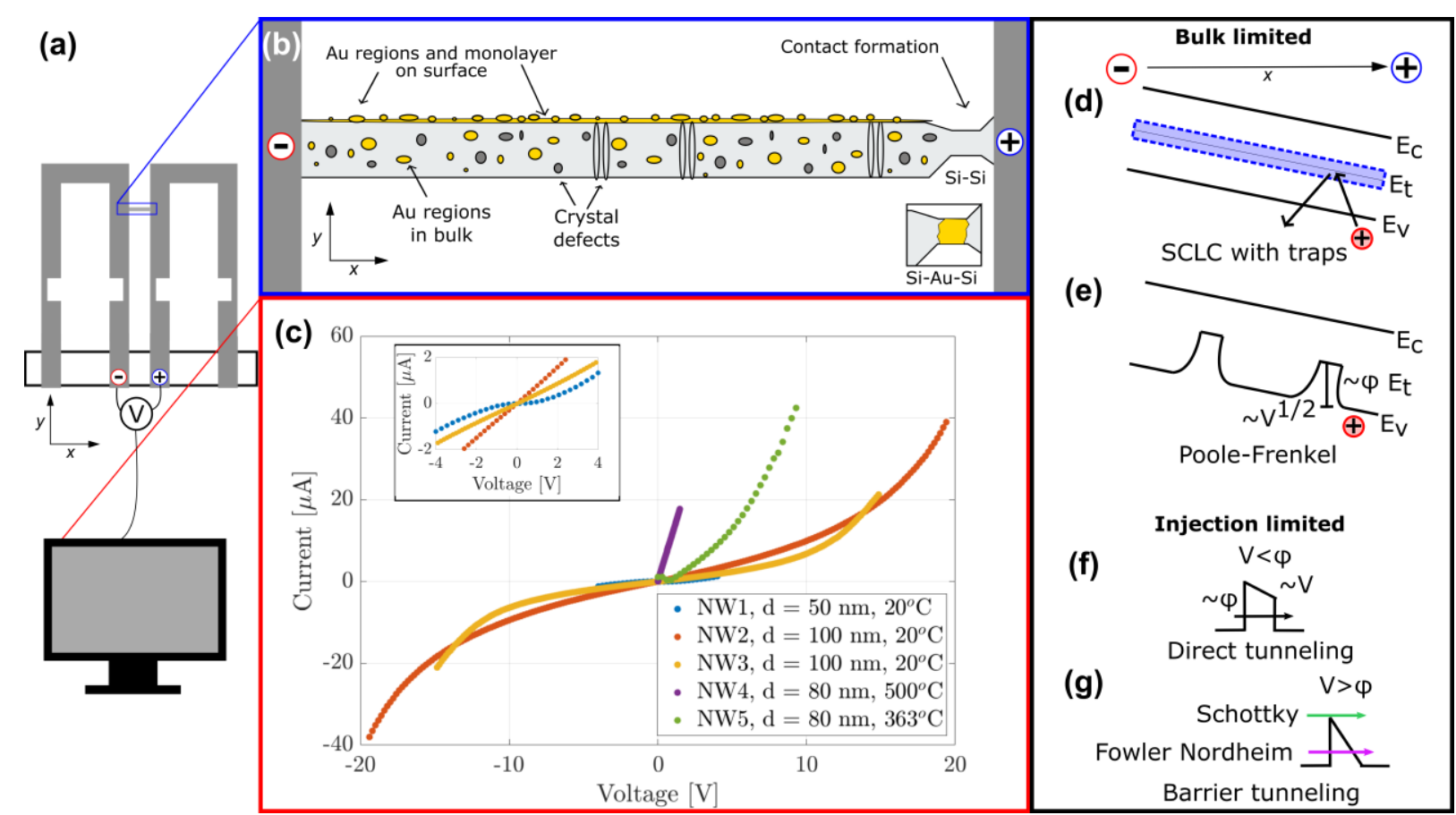

Figure 1. Illustration of setup up with (a) two cantilevers with a bridging nanowire, which has been (b) magnified to show different possible sources for nonlinear current. Growth is from left to right in the direction of $x$ axis. At the base is a Si-Si contact. At the tip, the contact may also be Si-Si or may include Au as shown in the inset. (c) $I$ - $V$ plots for five nanowires measured at various temperatures. (d)-(e) Energy bands for bulk-limited transport models and (f)-(g) energy barriers for injection-limited transport models that can produce nonlinear $I$ - $V$ characteristics, see text.

Table 1 - Transport model overview adapted from ref. [31] with resistive heating model from ref. [26].

\begin{tabular}{llllc}
\hline Model & Current-voltage relation & Ordinate & Abscissa & Mechanism \\
\hline \hline Ohmic (+ SCLC at low field) & $I \propto V$ & $I$ & $V$ & Bulk-limited \\
Ohmic + resistive heating & $I / V=1 / R_{o}+c_{c} I V$ & $I / V$ & $I V$ & Linear conductivity \\
Ohmic + resistive heating & $V / I=R_{o}+c_{r} I V$ & $V / I$ & $I V$ & Linear resistivity \\
SCLC (High field) & $I \propto V^{a}(a \geq 2)$ & $\ln (I)$ & $\ln (V)$ & Bulk-limited \\
Poole-Frenkel (PF) & $I \propto V \exp \left(a V^{1 / 2} / k T\right)$ & $\sinh ^{-1}(I / V)$ & $V^{1 / 2}$ & Bulk-limited \\
SCLC + PF & $I \propto V^{2} \sinh \left(a V^{1 / 2} / k T\right)$ & $\ln \left(I / V^{2}\right)$ & $V^{1 / 2}$ & Bulk-limited \\
Hill's law & $I \propto b \sinh (a V / k T)$ & $\sinh h^{-1}(I)$ & $V$ & Bulk-limited \\
Schottky emission & $I \propto \exp \left(a V^{1 / 2} / k T\right)$ & $\ln (I)$ & $V^{1 / 2}$ & Injection-limited \\
Fowler-Nordheim & $I \propto \mathrm{V}^{2} \exp (-a / V)$ & $\ln \left(I / V^{2}\right)$ & $1 / V$ & Injection-limited \\
\hline
\end{tabular}

The SCLC behaviour has previously been reported for silicon nanowires decorated by $\mathrm{TiO}_{2}$ nanoparticles [32], coaxial AlGaN/GaN nanowires [28] and InAs nanowires [33]. The two mechanisms, Ohmic and SCLC, are both bulk-limited mechanisms because the dominating conductance is via the bulk of the nanowire.

Another bulk-limited conductance model involves the PF effect, which is seen in materials with a large number of closely spaced defects or impurities. The carriers then reside on the defect sites and hop from one site to another, as illustrated in Figure 1(e) [34-36]. The PF model has a characteristic energy barrier $\varphi$ (Figure 1(e)), which is the energy needed to overcome the coulomb potential of the trap sites. If the defect sites are too far from each other, the hopping mechanism cannot keep up with the applied electric field $[35,37]$ resulting in a transport mechanism that combines 
(a)

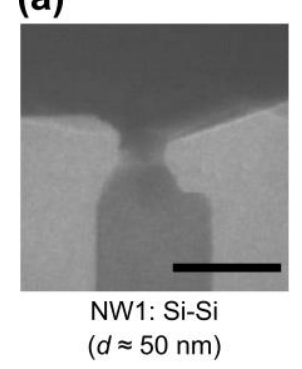

(b)

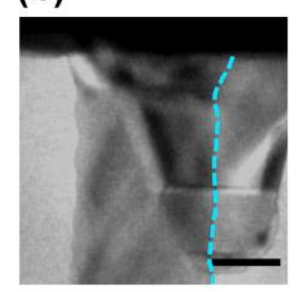

NW2: Si-Si

(c) $(\mathrm{d} \approx 100 \mathrm{~nm})$

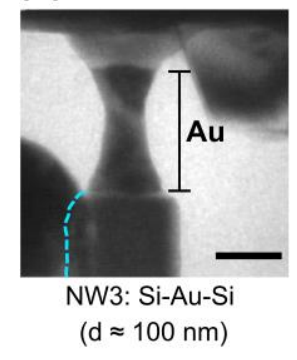

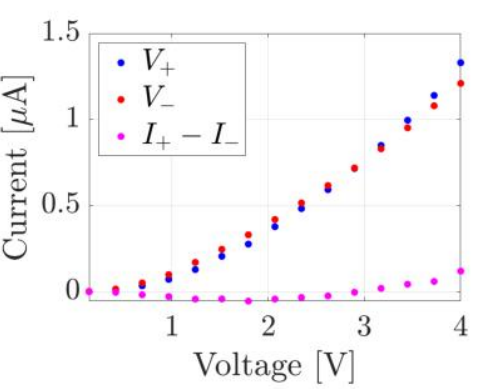
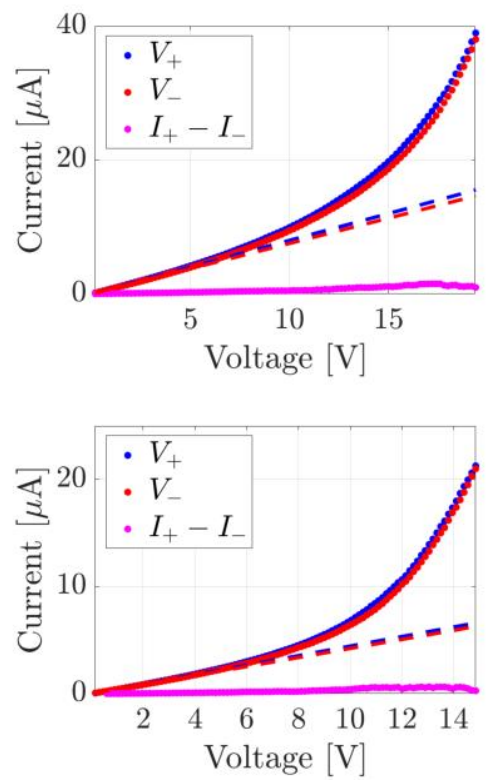

Figure 2. TEM images of three different types of nanowire connections: small diameter nanowires (a) and large diameter nanowires with (b) $\mathrm{Si}-\mathrm{Si}$ and (c) $\mathrm{Si}-\mathrm{Au}-\mathrm{Si}$ contacts, respectively. The corresponding $I-V$ curves are plotted with the absolute value of the negative voltage and current. The difference of the absolute value of positive and negative current $\left(I_{+}-I_{-}\right)$is also plotted. A linear Ohmic fit up to $3 \mathrm{~V}$ is illustrated for (b) and (c) by dashed lines. The blue lines in images (b) and (c) distinguish the nanowires of interest from overlapping out of focus wires. Scale bar is $50 \mathrm{~nm}$.

filling the traps with charge carriers and the hopping mechanism: in other words, a combination of SCLC and the $\mathrm{PF}$ effect. When the defect density is high, an overlap of the columbic potentials of the defect sites will lower the barrier of the carrier hopping, leading to transport defined by Hill's law $[35,38]$.

\subsection{Injection-limited current models}

The current can also be injection-limited, for instance, if the carriers pass through a metal-semiconductor interface [39]. The inset in Figure 1(b) shows a situation where nanowire growth was terminated in a way that creates a residual gold section with two gold-silicon interfaces. Furthermore, the

nanowire surface is also decorated by gold: growth is catalysed by an AuSi eutectic droplet which is known to leave up to a monolayer of gold on the sidewalls at the low disilane pressures used in the UHV-TEM [40,41]. The presence of a gold layer on the surface not only provides a possible transport path via the metal surface but could also affect transport through the bulk silicon nanowire through band bending leading to formation of a depletion region at a surface layer [42] as well as by the introduction of deep traps [41].

Another mechanism for injection-limited transport is thermionic emission (TE), involving only the carriers that have the energy to overcome the Schottky barrier. This contributes (Figure 1(g)) to the current flow at the $\mathrm{Au} / \mathrm{Si}$ interface at Au particles on the surface (or at the Au section in Figure 1(b) inset, if present). At low bias, the electrons cannot cross the barrier implying a high resistance, while at high bias, they can cross leading to a low differential resistance. TE is strongly temperature dependent, as higher temperature will increase the kinetic energy of the carriers [43]. TE leads to a rectifying effect causing an asymmetric $I-V$ behaviour when sweeping between positive and negative bias. However, for symmetric junctions as in the metal-semiconductor-metal configuration that we see experimentally, TE should produce a symmetric $I$ - $V$ curve $[44,45]$. TE transport has been reported for various examples of metal-semiconductor contacts such as $\mathrm{Au}$ nanoclusters on hexagonal silicon carbide [46] and Al-Ge nanowire heterostructures [47]. TE transport has also been reported for GaN nanowires decorated with Au nanoparticles that create local depletion regions in the semiconductor beneath each of the Au particles, thus inducing surface band banding and creating localized Schottky junctions on the surface of the nanowire [42]. There could be a similar situation for Au-decorated Si nanowires.

Field emission (FE), also known as Fowler-Nordheim (FN) injection, is similar to thermionic emission in the sense that it has an energy barrier that must be passed before the current can flow. However, instead of jumping over the energy barrier, the electrons tunnel through the (thin) barrier, as illustrated in Figure 1(g) [48]. A relevant study showing FN injection [49] involves conductance in thin gold films with separated grains that lead to the tunneling effect.

An overview of the $I-V$ curves expected for the different conduction models is summarised in Table 1 . With the ordinate and abscissa given in the table, straight-line plots should result if the $I-V$ data fits the model. In theory each model has a unique signature, while measurements often show mixed behaviour.

\section{Matching data to the models}

Since nanowires are grown and electrically connected inside the TEM under vacuum, they do not have an oxidised surface, gated substrate, or other sources of contamination that can come from exposure to ambient atmosphere. Hence, our 
experimental setup, unlike typical ex situ probing, allows us to measure $I-V$ curves on individual pristine, oxide-free nanowires with epitaxial contacts while simultaneously imaging the nanowire for signs of physical transformation or carrying out deliberate modification during the measurements.

However, TEM imaging requires irradiation of the nanowire by an electron beam, hence the influence of the beam on the in situ electrical measurements must be considered. Irradiation of the silicon nanowire by high-energy electrons can generate electron-hole pairs and trapped electrons that can charge insulating regions and interfaces. In principle (although not at the $10^{-10}$ Torr pressure range of the UHV-TEM), the beam can also generate radicals by radiolysis of trace amounts of gas present in the vacuum and these radicals can react with the nanowire surface. We investigated the influence of the beam by performing multiple measurements, summarised in Figure S3, on a nanowire with the beam on and off. We found no trend to indicate that beam exposure alters the transport properties of nanowires in our experiments.

\subsection{In situ electrical characterisation of pristine nanowire bridges}

The length of the nanowires is defined by the cantilever gap (approximately $2.5 \mu \mathrm{m}$ ), so the wire diameter, contact composition, and surface treatment are the experimental parameters that can be varied. The wires had resistances of a few $\mathrm{M} \Omega$, making the cantilever lead resistance (a few $\mathrm{k} \Omega$ ) negligible. The nanowire-to-cantilever spreading resistance $(\sim 20 \mathrm{k} \Omega)$ is also negligible [19]. Three different nanowires were characterised in this study. These are a small diameter nanowire (NW1) with a diameter of $\sim 50 \mathrm{~nm}$ and Si-Si contact (Figure 2(a)), and large-diameter nanowires with a diameter of approximately $100 \mathrm{~nm}$ and either a Si-Si contact (NW2), Figure 2(b), or a Si-Au-Si contact (NW3), Figure 2(c). The nanowire contact geometry is influenced by several factors [24] requiring careful control during the experiment since multiple wires can reach contact simultaneously. The nanowire diameter depends on the diameter of the eutectic droplet formed during the initial annealing of the thin gold film deposited on the cantilevers. Reproducibility is hence low, in the sense that diameter is not directly controlled, but this could in principle be improved by using size selected gold particles instead of a thin film.

\subsection{Model for small diameter nanowires}

We first discuss nanowire NW1, with a diameter of $\sim 50 \mathrm{~nm}$, that was connected at one end via a narrow neck created by electromigration of Au during Si-Si contact formation [24]. Figure 2(a) shows the structure and $I-V$ curve measured by sweeping from $-4 \mathrm{~V}$ to $4 \mathrm{~V}$. NW1 does not show an Ohmic behaviour. Joule heating effects can also be ignored as the temperature increase is below $10 \mathrm{~K}$ as shown in Figure S4. The analysis for the simple resistive heating model is given in Figure 3(a) (and Figure S5) and it does not show a linear behaviour of conductivity even for the low bias measurement, thus verifying that Joule heating is not responsible for the nonlinearity. The nonlinear behaviour must therefore come from other transport effects. The data are re-plotted with the four bulk-limited transport models in Figure 3(b) such that a straight line would show that the $I-V$ data fits one of the models. The corresponding fitting parameters and statistical results are summarised in Table $\mathrm{S} 1$ in Supporting Information. We observe straight line fits for both the SCLC and PF models, and a coefficient of the SCLC fit that is close to 2 corresponding to trap-free SCLC behaviour. However, as mentioned above, the nanowires grown in situ are expected to be covered with a surface layer of gold, introducing deep traps. For a thick nanowire with a diameter of $\sim 250 \mathrm{~nm}$, it has been argued that conduction is limited by bulk transport effects [50,51]. Considering a thinner wire, the deep traps introduced by the surface cannot be ignored so easily. Therefore, it is more likely that transport is dominated by a PF mechanism for the narrow nanowire. This conclusion is consistent with silicon nanowires examined elsewhere [50,51], although in that case bulk crystal defects were proposed to cause the traps instead. Crystal defects, like dislocations and stacking faults in the bulk, may contribute to the PF mechanism and can exist in conjunction with surface-induced deep traps. Our qualitative analysis shows that surface-induced traps are likely dominant: The $50 \mathrm{~nm}$ diameter NW1 and the larger $\sim 100 \mathrm{~nm}$ wires NW2 and NW3 were synthesised under similar VLS conditions but, as discussed in the following section, the larger NW2 and NW3 have linear $I-V$ characteristics below $|3 \mathrm{~V}|$. This difference in $I-V$ curves with respect to diameter hint at surface effects playing a role.

\subsection{Models for large diameter nanowires}

We now discuss the two wider nanowires, NW2 and NW3, with diameters close to $100 \mathrm{~nm}$ but with different contact structures. Both wires show $I-V$ curves with an Ohmic behavior for voltages smaller than $3 \mathrm{~V}$ (Figure 1(c) inset). This allows us to measure their resistance over this range as 1.25 2.5 M $\Omega$. The fitting parameters and statistical results of the $I$ $V$ curve fits are summarised in Table $\mathrm{S} 2$ and Figure S7. Nanowires grown in the UHV-TEM are expected to be unintentionally p-doped, according to Ref. [19], with p-dopant level estimated to be in the range of $10^{15}-10^{16} \mathrm{~cm}^{-3}$ [19]. Hence, the nanowires will form a $\mathrm{p}^{+}-\mathrm{p}-\mathrm{p}^{+}$junction with the $\mathrm{p}^{+}$ doped cantilevers that are boron doped to $3 \times 10^{17} \mathrm{~cm}^{-3}$.

The dopant atoms in the nanowires may come from impurities in the disilane (typically such gases have trace amounts of diborane) and/or from the doped cantilever heater at the initiation of growth. 


\section{Resistive heating}

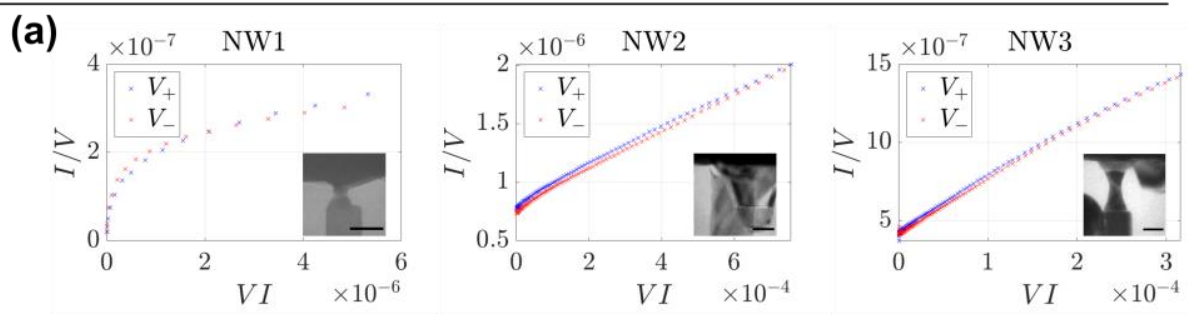

\section{Bulk limited}
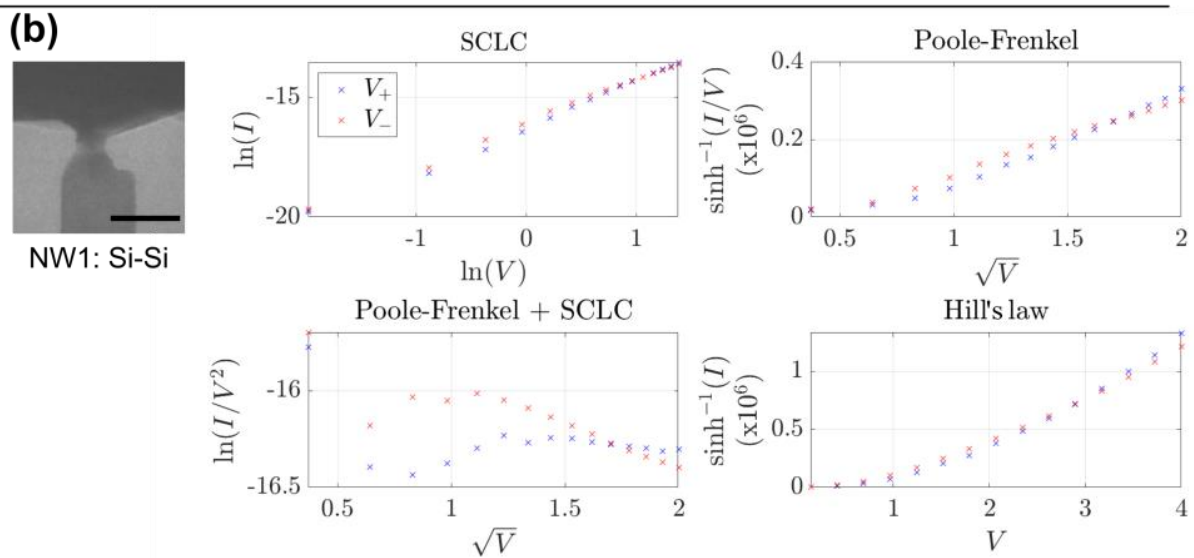

(c)
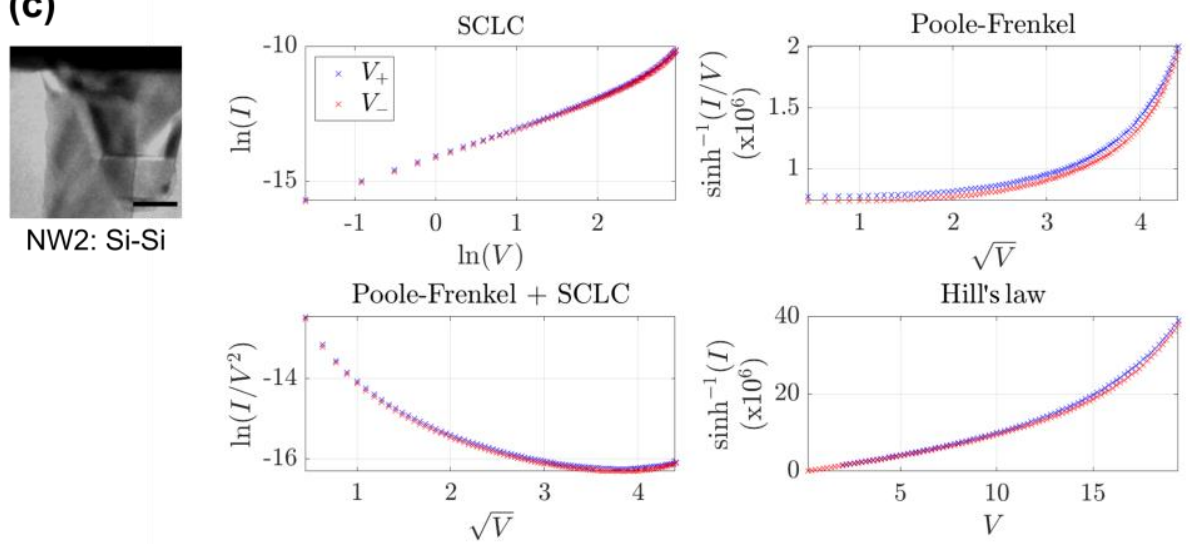

Injection limited

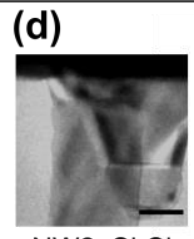

NW2: Si-Si

(e)

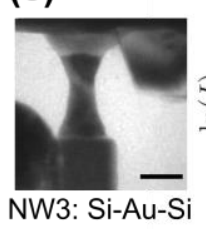

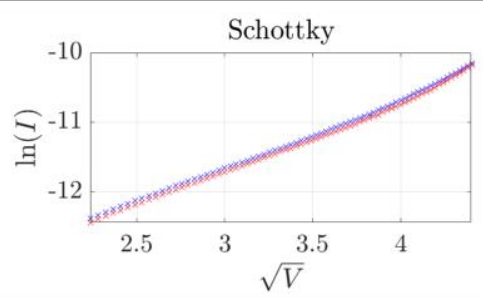

Schottky

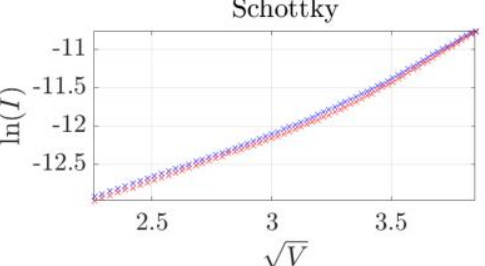

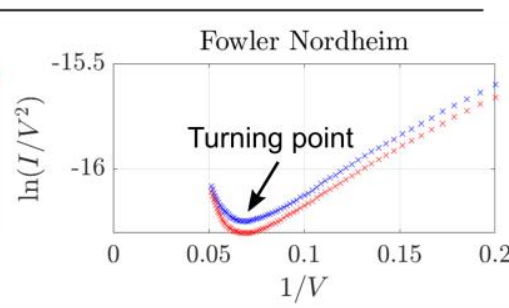

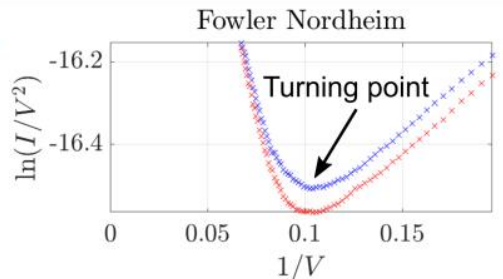

Figure $3 I-V$-measurements for nanowires NW1, NW2 and NW3 with different transport models applied. (a) Resistive heating model applied on I- $V$ curves from NW1, NW2 and NW3. (b)-(c) Bulk-limited current models given for nanowires with two different diameters, both with Si-Si contacts. (d)-(e) Injection-limited current models for two kinds of contacts, $\mathrm{Si}-\mathrm{Si}$ and $\mathrm{Si}-\mathrm{Au}-\mathrm{Si}$. The scale bar in all TEM images is $50 \mathrm{~nm}$. 
(a)

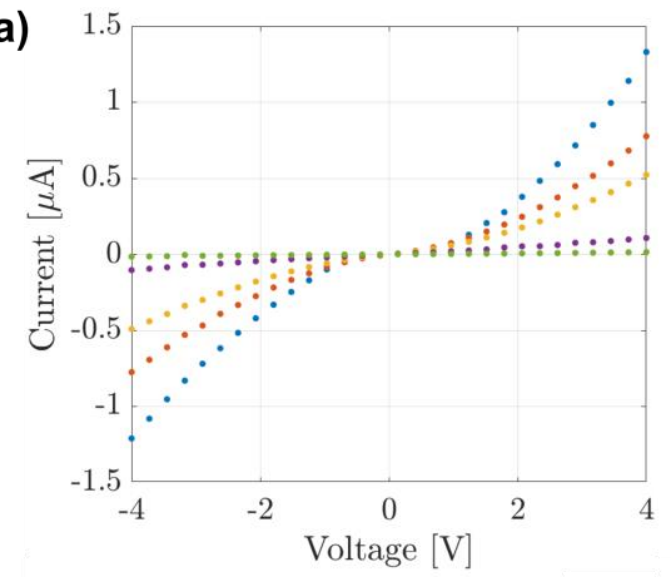

Step 0: Before oxidation

Step 1: in-situ TEM oxidation (13 min

Step 2: in-situ TEM oxidation (40 $\mathrm{min}$

Step 3: in-situ TEM oxidation $\left(400^{\circ} \mathrm{C}, 35 \mathrm{~min}\right)$

Step 4: ex-situ TEM oxidation (10 min)

(c)

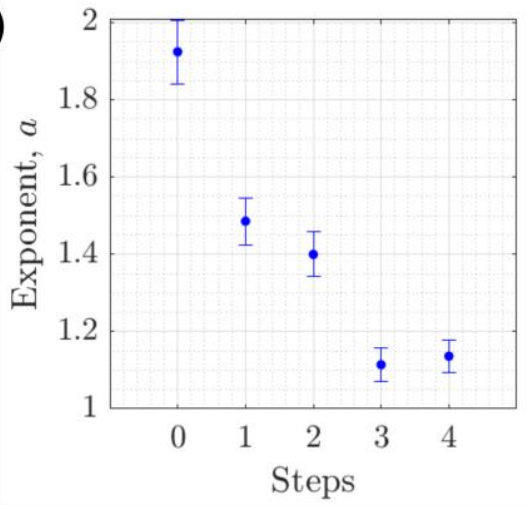

(b)

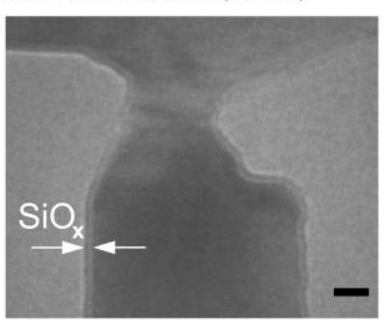

(d)

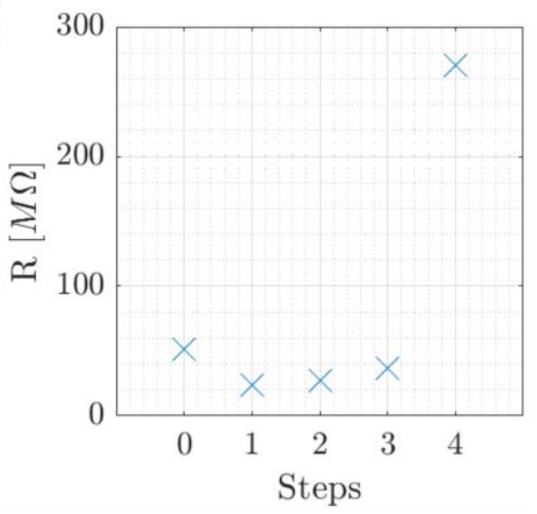

Figure 4 - Oxidation of nanowire NW1 in four different steps. (a)The $I$ - $V$ curves have been fitted to a power law $\left(I \propto V^{a}\right)$ and the exponent for the different steps are given in (c) together with the changes in resistance in (d). The TEM image (b) is after ex situ oxidation. Scale bar in (b) is $10 \mathrm{~nm}$.

This can occur because the AuSi droplet initially forms a eutectic melt with Si from the substrate (cantilever) and some dopant atoms from the substrate may therefore end up in the nanowire. The resistance reported above corresponds to an effective hole concentration of $2-4 \times 10^{16} \mathrm{~cm}^{-3}$, assuming the mobility is constant at $450 \mathrm{~cm}^{2} / \mathrm{Vs}$ and the nanowires are pristine [52]. This can be compared to the doping of nanowires grown under similar conditions. Kimukin et al. [53] studied intentionally doped but oxide-free gold catalysed VLS silicon nanowires of similar diameter grown in a chemical vapour deposition chamber in which no surface layer of gold is expected because of the higher silane pressure. These nanowires showed a resistance of $600 \mathrm{k} \Omega$, which, correcting for length, corresponds to $250 \mathrm{k} \Omega$. Hence, the resistance of our unintentionally doped NWs is larger by a factor $\sim 4$ than reported literature. Dopants can in principle be measured by holographic methods [54], but given the complexity of having $\mathrm{Au}$ on the surface, it may be difficult to achieve reliable results.

A high temperature $\left(500 \pm 10^{\circ} \mathrm{C}\right)$ measurement in the range $0 \mathrm{~V}$ to $-1.5 \mathrm{~V}$ was also obtained for another nanowire (NW4) and agrees well with an Ohmic fit (Figure S8 and S9) giving a resistance of $0.08 \mathrm{M} \Omega$. The decrease in the resistance at high temperatures is by a factor of 25 compared to the room present. The temperature increase from resistive heating temperature measurements. A final nanowire (NW5) measured at the eutectic temperature of $363^{\circ} \mathrm{C}$ (Figure 1(c), S8 and S10) also shows Ohmic behaviour at lower voltages with a resistance of approximately $0.37 \mathrm{M} \Omega$. The reduction in resistance at increasing temperatures may be explained by a change in the dominant carriers. At higher temperatures, the sample may become intrinsic and the electrons have higher mobility than that of the extrinsic holes. At $500^{\circ} \mathrm{C}$, the intrinsic carriers will dominate the bulk charge transport [55].

Above $3 \mathrm{~V}$, nanowires NW2 and NW3 both exhibit nonlinear behaviour. The temperature profile is likely to be important under these conditions. For NW2, the power used is up to $0.8 \mathrm{~mW}$, resulting in an expected temperature increase of almost $250 \mathrm{~K}$ at the center of the wire as shown in Figure $\mathrm{S} 4(\mathrm{~b})$. For NW3, greater than $100 \mathrm{~K}$ rise is expected at the center (Figure S4(c)). Both wires show linear plots of conductivity with power (Figure 3(a)), indicating that a Joule heating effect is present. The temperature increase from resistive heating will have an impact on Ohmic transport, but it will also affect the different PF models and Schottky emission - although not the FN effect. The following analysis 
should therefore be taken with caution since temperature affects most bulk transport models. To avoid effects from a transition region between competing models in a parallel circuit of the bulk nanowire and its surface, we apply the models above $5 \mathrm{~V}$ where the nonlinear behavior is clear. We are thereby assuming a series circuit via either the nanowire or its surface, with just one transport model being dominant.

The two large diameter nanowires, NW2 and NW3, do not indicate SCLC behaviour as their exponent is $<2$, as reported in Table S1. Nor do they fit the PF and Hill's laws, as illustrated in Figure 3(c) and Figure S6. The poor fits indicate that the behaviour at higher voltages is not dominated by the bulk-limited models. Hence, at high voltages we consider injection-limited currents that arise from gold on the surface of the nanowires. (Only NW3 has a Si-Au-Si region so this cannot explain both sets of results.) We consider the effects on transport $[42,49,56,57]$ of either $\sim 1$ monolayer of gold on the surface, as expected for nanowires [40], or small $(5-10 \mathrm{~nm})$ gold islands, also known to form during contact [19]. The TEM resolution in our experiments does not allow for confirming the presence of the exact surface coverage, but TEM of oxidised wires (Figure S11) does indicate the presence of gold in some form on the nanowire surface, as described below.

Gold on the surface can bend the semiconductor energy bands [42], which can lead to thermionic emission for a semiconductor at high voltages. Another source for injectionlimited conductance is if current passes across the surface via the Au layer at high voltages. 1 monolayer of gold can have sufficient separation between the gold atoms that an injectionlimited current will appear [49]. In the case of lower gold coverage, the separation of individual gold atoms leads to tunneling.

As was discussed in Table 1, TE will produce a straight line when plotting $\ln (I)$ vs $V^{1 / 2}$, while $\mathrm{FN}$ emission will give a turning point at a characteristic voltage when plotting $\ln \left(I / V^{2}\right)$ vs. $1 / V$ with a negative slope for increasing $1 / V$ (decreasing voltages). Below the barrier, the curve will have a logarithmic growth. The characteristic turning point from FN emission is indeed visible for both NW2 and NW3, i.e. the Si-Si and Si$\mathrm{Au}-\mathrm{Si}$ contacts, in Figure 3(d),(e). Similar behaviour is seen for NW5 at $363^{\circ} \mathrm{C}$ as illustrated in Figure S10. However, at high voltages, the slope is not negative but rather positive, which indicates that transport is not by FN tunneling via the surface layer at high temperature [58].

It is worth noting in Figure 2 an increase in recorded current for one voltage sweep direction compared to the other as the voltage gets near the energy barrier (15 V and $10 \mathrm{~V}$ for NW2 and NW3). The voltages are applied from zero to the negative maximum and then from zero to the positive maximum. The current is noticeably higher for the latter measurement, indicating that some phenomenon has happened during the first voltage sweep such as charging of impurities or defects that results in this hysteresis. The hysteresis may be due to traps on the surface or in the bulk that are charged the second time voltage is applied, or it may instead be due to restructuring of the gold due to electromigration [24].

\subsection{Influence of nanowire surface oxidation}

Different gases can be introduced in the TEM column, allowing us to modify the composition of the nanowire surface between $I-V$ measurements. To study the effect of oxidation we introduced oxygen after measuring $I$ - $V$ data on oxide-free nanowire NW1, Figure 4. Pressures in the $10 \mu$ Torr range were used for each of three in situ oxidation steps, and the last step included resistively heating the sample to approximately $400^{\circ} \mathrm{C}$. This is expected to enhance the surface oxidation, since the reaction is strongly temperature dependent [59]. A final oxidation step was carried out at 1 atm. ex situ by removing the sample from the TEM vacuum for 10 minutes. No oxidation layer was visible in the TEM before this ex situ oxidation, while an oxide layer of $\sim 2.4 \mathrm{~nm}$ was visible afterwards (Figure 4(b) and Figure S11(b)). A linear fit of $I$ - $V$ data of the last two oxidation steps (Table S4) shows an increase in the resistance by a factor of 10 indicating that the oxide layer affects the resistance strongly beyond the minuscule reduction in the diameter of Si from the oxidation. The resistance values for each oxidation step are summarised in Figure 4(d).

These curves were fitted with a power law and the exponent of the power law for each step is shown in Figure 4(c). This clearly shows a shift from a power law with an exponent of 2 , indicating the PF model as discussed above, down to 1.5. After annealing, the exponent goes down to 1 . The surface modification removes the nonlinearity, showing the effect of the surface on the conductance; annealing leads to linear behaviour. We speculate that this may be due to changes in the traps, perhaps by breaking up the continuous surface layer of $\mathrm{Au}$ into isolated $\mathrm{Au}$ islands, as shown in Figure S11. This result could therefore indicate that the initial nonlinearity was caused by a PF trapping effect from the gold layer rather than the SCLC trap-free effect, as we have argued above. In addition to breaking up the continuous Au layer, the drastic decrease in the nanowire conductance might also be attributed to the formation of a $\mathrm{Si} / \mathrm{SiO}_{2}$ interface. The presence of interface states on the wire surface and trapped charges in the native oxide may create a depletion region below the oxide layer thereby decreasing the effective conducting area and effective hole concentration [60]. Further, the dielectric mismatch will increase the donor ionization energy resulting in dopant deactivation [61]. 
Table 2 - Summary of results

\begin{tabular}{llllll}
\hline Nanowire & Contact & $\mathbf{d}(\mathbf{n m})$ & $\mathbf{T}\left({ }^{\circ} \mathbf{C}\right)$ & $\boldsymbol{I}-\boldsymbol{V}$ curve & Best Model \\
\hline \hline $\mathrm{NW} 1$ & $\mathrm{Si}-\mathrm{Si}$ & 50 & $\mathrm{RT}$ & nonlinear & Poole-Frenkel \\
$\mathrm{NW} 1+\mathrm{SiO}_{2}$ & $\mathrm{Si}-\mathrm{Si}$ & 50 & $\mathrm{RT}$ & linear & Ohmic \\
$\mathrm{NW} 2<|3 \mathrm{~V}|$ & $\mathrm{Si}-\mathrm{Si}$ & 100 & $\mathrm{RT}$ & linear & Ohmic \\
$\mathrm{NW} 2>|5 \mathrm{~V}|$ & $\mathrm{Si}-\mathrm{Si}$ & 100 & $\mathrm{RT}$ & nonlinear & Resistive heating / Fowler- Nordheim \\
$\mathrm{NW} 3<|3 \mathrm{~V}|$ & $\mathrm{Si}-\mathrm{Au}-\mathrm{Si}$ & 100 & $\mathrm{RT}$ & linear & Ohmic \\
$\mathrm{NW} 3>|5 \mathrm{~V}|$ & $\mathrm{Si}-\mathrm{Au}-\mathrm{Si}$ & 100 & $\mathrm{RT}$ & nonlinear & Resistive heating / Fowler- Nordheim \\
$\mathrm{NW} 4$ & $\mathrm{Si}-\mathrm{Si}$ & 80 & 500 & linear & Ohmic \\
$\mathrm{NW5}<|3 \mathrm{~V}|$ & $\mathrm{Si}-\mathrm{Si}$ & 80 & 363 & linear & Ohmic \\
$\mathrm{NW} 5>|5 \mathrm{~V}|$ & $\mathrm{Si}-\mathrm{Si}$ & 80 & 363 & nonlinear & Resistive heating / Fowler-Nordheim \\
\hline
\end{tabular}

Hence, PF trapping by the gold layer and the $\mathrm{Si} / \mathrm{SiO}_{2}$ interface effects due to oxidation in ambient can account for the changes in the conductance.

\subsection{Higher voltages for oxidised nanowires}

After the fourth oxidation step, high voltages were applied to NW1. The nanowire was able to survive up to $35 \mathrm{~V}$ in vacuum (Figure S12). The $I-V$ measurements show a characteristic avalanche breakdown current with a low dynamic resistance above the threshold voltage, as seen in previous studies of $\mathrm{Si}-\mathrm{Au}-\mathrm{Si}$ connected nanowires [19]. The avalanche threshold voltages are expected to be above $20 \mathrm{~V}$ (Table S5) based on the model discussed by Kallesøe et al. [19], as shown in Figure S12.

\subsection{Temperature effect on oxidised nanowires}

We systematically studied the temperature dependence of the $I-V$ curves of two nanowires, NW6 and NW7, oxidised in ambient atmosphere and probed using ex situ TEM on a heating stage to study the thermoresistivity of the nanowires and extract the related activation energy. We found the $I-V$ curves to be linear (Figure S13). The Arrhenius plot, Figure $\mathrm{S} 14$, of the temperature-dependent resistance of ambient oxidised nanowires shows an Arrhenius dependence with activation energy $E_{a}$ of the order $300-350 \mathrm{meV}$ (Table S7). This is considerably more than the $60 \mathrm{meV}$ expected [56] for hopping conduction between gold islands. It is also higher than the $60 \mathrm{meV}$ reported for detrapping from Coloumb traps that originate from crystallographic misorientations [50,51]. The high activation energy is similar to that found from deep traps caused by Au within and on the sidewalls of highly pdoped silicon nanowires studied by deep-level and photoinduced current transient spectroscopy [62]. In these nanowires, the electrical conductance was also affected by deep traps from gold in nanowires with a monolayer of gold on the surface, but was more clearly measurable when the surface was oxidised.

\section{Conclusion}

We have investigated transport mechanisms in individual silicon nanowires (with $\mathrm{Au}$ on the surface) that were grown, electrically connected and modified inside a TEM. In this setup, nanowires can be probed at high temperatures as they are connected between microheaters. In addition to manipulating transport by temperature, we can also introduce gases like oxygen to oxidise the wire surface inside the TEM.

The observed $I-V$ curves of nanowires before and after oxidation and at room and high temperature were compared to several possible transport models, with those most consistent with the data summarized in Table 2 . The analysis using a wide range of conduction models also serves as a review of the variety of conduction mechanisms possible in nanowire devices. We find that no single model can explain all the data, suggesting that conclusive model assignment in nanowires requires a range of experiments with varied parameters such as radius, doping and temperature. However, from the available data, we observed that electronic transport in wires of different diameter is dissimilar despite the common growth mechanism. The electronic transport in $\sim 50 \mathrm{~nm}$ diameter nanowires is nonlinear down to $0.13 \mathrm{~V}$ and appears best described by the Poole-Frenkel effect, while larger wires with $100 \mathrm{~nm}$ diameter exhibited Ohmic behaviour at low voltages and nonlinear behaviour above $5 \mathrm{~V}$ that fits with resistive heating models and also shows the characteristic turning point from the injection-limited Fowler-Nordheim model. We demonstrated a transition from nonlinear to linear $I-V$ characteristics by progressive in situ and ambient oxidation of the nanowire surface. TEM images show a modification of the surface structure as oxidation breaks the continuous gold layer on the surface into gold islands, thus reducing the conduction on the surface. The increase (by a factor of 10) in the resistance of the nanowire after oxidation could also hint at the formation of oxide traps that lead to a depletion effect. Transition to linear $I-V$ behavior is also achieved by operating the nanowire at high temperatures around $500^{\circ} \mathrm{C}$, and the dominant contribution to transport then comes from intrinsic carriers. For nanowires oxidised in atmosphere, increasing the 
temperature reveals an activation energy of $300 \mathrm{meV}$ that further hints at formation of deep trap states in oxidised wires.

Our experiments demonstrate that in situ electrical measurements can reveal transitions in nanowire conduction mechanisms that would be challenging tasks for postfabrication, ex situ characterisation tools. Using in situ TEM, we can measure the precise dimensions of the electrically probed nanostructure and combine in situ data with techniques exclusive to TEM such as HRTEM, electron diffraction, EDS and EELS. A comprehensive relation between structure, composition and electronic transport can be established, provided enough data is collected, with measurements at a range of temperatures, diameters and surface structures. Finally, the development of this in situ method to grow and electrically characterise silicon nanowires inside the TEM can help us in understanding the behaviour of other nanowire compositions, potentially including III-V nanowires, heterostructure devices and quantum dot systems. In the future, in addition to in situ heating or surface modification, stimuli like irradiation with light could also be used to perform detailed characterisation of optically active nanowires. Further, TEM holder setups can be developed to accommodate operational nanowire devices inside the TEM, thus linking nanowire structure to device operation in real-time.

\section{Acknowledgements}

This work was part of the FTP Nano Live project, which was funded by The Danish Council for Independent Research (Case No.10-083797). This work was partly supported by The Danish National Research Foundation's Center for Individual Nanoparticle Functionality, CINF (DNRF54). The work was also supported by DTU Nanolab, DTU Fotonik and the Knut and Alice Wallenberg Foundation.

\section{Author contributions}

O. These authors contributed equally to this work.

\section{References}

[1] Cui Y, Zhong Z, Wang D, Wang W U and Lieber C M 2003 High Performance Silicon Nanowire Field Effect Transistors Nano Lett. 3 149-52

[2] Weber W M, Geelhaar L, Graham A P, Unger E, Duesberg G S, Liebau M, Pamler W, Chèze C, Riechert H, Lugli P and Kreupl F 2006 Silicon-Nanowire Transistors with Intruded Nickel-Silicide Contacts Nano Lett. 62660 6

[3] Czaban J A, Thompson D A and LaPierre R R 2009 GaAs Core-Shell Nanowires for Photovoltaic Applications Nano Lett. 9 148-54

[4] Krogstrup P, Jørgensen H I, Heiss M, Demichel O, Holm J V., Aagesen M, Nygard J and Fontcuberta I Morral A 2013 Single-nanowire solar cells beyond the Shockley-Queisser limit Nat. Photonics 7 306-10 5

Koester R, Sager D, Quitsch W-A, Pfingsten O, Poloczek A, Blumenthal S, Keller G, Prost W, Bacher G and Tegude F-J 2015 High-Speed GaN/GaInN Nanowire Array LightEmitting Diode on Silicon(111) Nano Lett. 15 2318-23

[7] Breuer S, Pfüller C, Flissikowski T, Brandt O, Grahn H T, Geelhaar L and Riechert H 2011 Suitability of $\mathrm{Au}$ - and Self-Assisted GaAs Nanowires for Optoelectronic Applications Nano Lett. 11 1276-9

[8] Zheng G, Patolsky F, Cui Y, Wang W U and Lieber C M 2005 Multiplexed electrical detection of cancer markers with nanowire sensor arrays Nat. Biotechnol. 23 1294-301

[9] Stern E, Klemic J F, Routenberg D A, Wyrembak P N, Turner-Evans D B, Hamilton A D, LaVan D A, Fahmy T M and Reed M A 2007 Label-free immunodetection with CMOS-compatible semiconducting nanowires Nature 445 519-22

[10] Demontis V, Rocci M, Donarelli M, Maiti R, Zannier V, Beltram F, Sorba L, Roddaro S, Rossella F and Baratto C 2019 Conductometric Sensing with Individual InAs Nanowires Sensors 192994

[11] Kong J, Franklin N R, Zhou C, Chapline M G, Peng S, Cho K and Dai H 2000 Nanotube molecular wires as chemical sensors Science (80-. ). 287 622-5

[12] Yuan G D, Zhou Y B, Guo C S, Zhang W J, Tang Y B, Li Y Q, Chen Z H, He Z B, Zhang X J, Wang P F, Bello I, Zhang R Q, Lee C S and Lee S T 2010 Tunable electrical properties of silicon nanowires via surface-ambient chemistry ACS Nano 4 3045-52

[13] Amato M and Rurali R 2016 Surface physics of semiconducting nanowires Prog. Surf. Sci. 91 1-28

[14] Chen X, Wong C K Y, Yuan C A and Zhang G 2013 Nanowire-based gas sensors Sensors Actuators, B Chem. 177 178-95

[15] Ramgir N S, Yang Y and Zacharias M 2010 Nanowirebased sensors Small 6 1705-22

[16] Patolsky F and Lieber C M 2005 Nanowire nanosensors Mater. Today 8 20-8

[17] Patolsky F, Zheng G and Lieber C M 2006 Nanowirebased biosensors Anal. Chem. 78 4260-9

[18] Ross F M 2010 Controlling nanowire structures through real time growth studies Reports Prog. Phys. 73114501

[19] Kallesøe C, Wen C Y, Booth T J, Hansen O, Bøggild P, Ross F M and Mølhave K 2012 In situ TEM creation and electrical characterization of nanowire devices Nano Lett. 12 2965-70

[20] Nam S W, Chung H S, Lo Y C, Qi L, Li J, Lu Y, Johnson A T C, Jung Y, Nukala P and Agarwal R 2012 Electrical wind force-driven and dislocation-templated amorphization in phase-change nanowires Science (80-. ). 336 1561-6

[21] Chiu C H, Huang C W, Chen J Y, Huang Y T, Hu J C, Chen L T, Hsin C L and Wu W W 2013 Copper silicide/silicon nanowire heterostructures: In situ TEM observation of growth behaviors and electron transport properties Nanoscale 5 5086-92

[22] Canepa S, Alam S B, Ngo D-T, Ross F M and Mølhave K 2016 In Situ TEM Electrical Measurements Controlled Atmosphere Transmission Electron Microscopy (Springer International Publishing) pp 281-300

[23] Kallesøe C, Wen C Y, Mølhave K, Bøggild P and Ross F M 2010 Measurement of Local Si-Nanowire Growth Kinetics Using in situ Transmission Electron Microscopy of Heated Cantilevers Small 6 2058-64 
[24] Alam S B, Panciera F, Hansen O, Mølhave K and Ross F M 2015 Creating New VLS Silicon Nanowire Contact Geometries by Controlling Catalyst Migration Nano Lett. 15 6535-41

[25] Panciera F, Norton M M, Alam S B, Hofmann S, Mølhave $\mathrm{K}$ and Ross F M 2016 Controlling nanowire growth through electric field-induced deformation of the catalyst droplet Nat. Commun. 712271

[26] Mølhave K, Gudnason S B, Pedersen A T, Clausen C H, Horsewell A and Bøggild P 2006 Transmission electron microscopy study of individual carbon nanotube breakdown caused by joule heating in air Nano Lett. 6 $1663-8$

[27] Lampert M A 1956 Theory of Space-Charge-Limited Currents in an Insulator with Traps Phys. Rev. 103 1648-

[28] Simpkins B S, Mastro M A, Eddy C R, Hite J K and Pehrsson P E 2011 Space-charge-limited currents and trap characterization in coaxial AlGaN/GaN nanowires J. Appl. Phys. 11044303

[29] Mott N F and Gurney R W 1940 Electronic processes in ionic crystals Electron. Process. Ion. Cryst.

[30] Mark P and Helfrich W 1962 Space-Charge-Limited Currents in Organic Crystals J. Appl. Phys. 33 205-15

[31] Gonon P, Deneuville A, Fontaine F and Gheeraert E 1995 Electrical conduction and deep levels in polycrystalline diamond films J. Appl. Phys. 78 6633-8

[32] Rasool K, Rafiq M A, Ahmad M, Imran Z, Batool S S, Nazir A, Durrani Z A K and Hasan M M 2015 Charge injection and trapping in $\mathrm{TiO} 2$ nanoparticles decorated silicon nanowires arrays Appl. Phys. Lett 10673101

[33] Alagha S, Shik A, Ruda H E, Saveliev I, Kavanagh K L and Watkins S P 2017 Space-charge-limited current in nanowires J. Appl. Phys. 121174301

[34] Frenkel J 1938 On Pre-Breakdown Phenomena in Insulators and Electronic Semi-Conductors Phys. Rev. 54 647-8

[35] May P W, Höhn S, Ashfold M N R, Wang W N, Fox N A, Davis T J and Steeds J W 1998 Field emission from chemical vapor deposited diamond and diamond-like carbon films: Investigations of surface damage and conduction mechanisms J. Appl. Phys. 84 1618-25

[36] Sze S M 2006 Physics of Semiconductor Devices: Third Edition (John Wiley and Sons)

[37] Murgatroyd P N 1970 Theory of space-charge-limited current enhanced by Frenkel effect J. Phys. D. Appl. Phys. 3308

[38] Hill R M 1971 Poole-Frenkel conduction in amorphous solids Philos. Mag. 23 59-86

[39] Chiang T H and Wager J F 2018 Electronic conduction mechanisms in insulators IEEE Trans. Electron Devices $\mathbf{6 5}$ $223-30$

[40] Hannon J B, Kodambaka S, Ross F M and Tromp R M 2006 The influence of the surface migration of gold on the growth of silicon nanowires Nature 440 69-71

[41] Den Hertog M I, Rouviere J-L, Dhalluin F, Desré P J, Gentile P, Ferret P, Oehler F and Baron T 2008 Control of Gold Surface Diffusion on Si Nanowires Nano Lett 8 $1544-50$

[42] Sundararajan J P and McIlroy D N 2011 Electron transport properties of individual gallium nitride nanowire decorated with gold nanoparticles Proc. IEEE Conf. Nanotechnol. $1331-4$

[43] Zhang Z Y, Jin C H, Liang X L, Chen Q and Peng L M 2006 Current-voltage characteristics and parameter retrieval of semiconducting nanowires Appl. Phys. Lett. 88 039901

[44] Sze S M, Coleman D J and Loya A 1971 Current transport in metal-semiconductor-metal (MSM) structures Solid State Electron. 14 1209-18

[45] Elhadidy H, Sikula J and Franc J 2012 Symmetrical current-voltage characteristic of a metal-semiconductormetal structure of Schottky contacts and parameter retrieval of a CdTe structure Semicond. Sci. Technol. 27 015006

[46] Ruffino F, Grimaldi M G, Giannazzo F, Roccaforte F and Raineri V 2006 Size-dependent Schottky Barrier Height in self-assembled gold nanoparticles Appl. Phys. Lett. 89 243113

[47] Kral S, Zeiner C, Stöger-Pollach M, Bertagnolli E, Den Hertog M I, Lopez-Haro M, Robin E, El Hajraoui K and Lugstein A 2015 Abrupt Schottky Junctions in Al/Ge Nanowire Heterostructures Nano Lett. 15 4783-7

[48] Fowler R and Nordheim L 1928 Electron emission in intense electric fields Proc. R. Soc. London Ser. Acontaining Pap. a Math. Phys. Character 119

[49] Alkhatib A, Souier T and Chiesa M 2011 Morphology dependent electrical transport behavior in gold nanostructures Thin Solid Films 520 656-61

[50] Acharya S and Kottantharayil A 2018 Poole-frenkel transport in gold catalyzed VLS grown silicon nanowires IEEE Trans. Electron Devices 65 1685-91

[51] Acharya S, Tongbram B, Samajdar I S and Kottantharayil A 2020 What causes Poole-Frenkel transport in VLS grown silicon nanowires? Mater. Sci. Semicond. Process. 105104749

[52] Jacoboni C, Canali C, Ottaviani G and Alberigi Quaranta A 1977 A review of some charge transport properties of silicon Solid State Electron. 20 77-89

[53] Kimukin I, Islam M S and Williams R S 2006 Surface depletion thickness of $\mathrm{p}$-doped silicon nanowires grown using metal-catalysed chemical vapour deposition Nanotechnology 17 S240-5

[54] Den Hertog M I, Schmid H, Cooper D, Rouviere J L Björk M T, Riel H, Rivallin P, Karg S and Riess W 2009 Mapping active dopants in single silicon nanowires using off-axis electron holography Nano Lett. 9 3837-43

[55] Green M A 1990 Intrinsic concentration, effective densities of states, and effective mass in silicon J. Appl. Phys. 67 2944-54

[56] Zhu X, Hasko D G, Hofmann S and Milne W I 2011 Temperature dependent resistance and cryogenic microwave spectroscopy of Au decorated silicon nanowires J. Appl. Phys. 109113713

[57] Shekhar S, Anjia L, Matsui H and Khondaker S I 2011 Electrical transport properties of peptide nanotubes coated with gold nanoparticles via peptide-induced biomineralization Nanotechnology 22 95202-6

[58] Pananakakis G, Ghibaudo G, Kies R and Papadas C 1995 Temperature dependence of the Fowler-Nordheim current in metal-oxide-degenerate semiconductor structures $J$. Appl. Phys. 78 2635-41

[59] Seo K, Sharma S, Yasseri A A, Stewart D R and Kamins T I 2006 Surface Charge Density of Unpassivated and Passivated Metal-Catalyzed Silicon Nanowires Electrochem. Solid-State Lett. 9 G69

[60] Jie J, Zhang W, Peng K, Yuan G, Lee C S and Lee S T 2008 Surface-dominated transport properties of silicon nanowires Adv. Funct. Mater. 18 3251-7 
[61] Björk M T, Schmid H, Knoch J, Riel H and Riess W 2009 Donor deactivation in silicon nanostructures Nat.

Nanotechnol. 4 103-7

[62] Sato K, Castaldini A, Fukata N and Cavallini A 2012

Electronic level scheme in boron- and phosphorus-doped silicon nanowires Nano Lett. 12 3012-7 